\title{
Análise dos fatores facilitadores e obstáculos para a implementação da gestão do risco empresarial no Brasil - Uma perspectiva de mudanças
}

Analysis of facilitating factors and obstacles for the implementation of business risk management in Brazil - A perspective of changes

Análisis de factores facilitadores y obstáculos para la implementación de la gestión de riesgos empresariales en Brasil: Una perspectiva de câmbios

\section{Resumo}

A utilização de um sistema de gerenciamento de riscos é um dos aspectos imprescindíveis para a sobrevivência de empresas do setor de construção, sobretudo em tempos pós crise. No entanto, o processo de implementação desse sistema requer mudanças no contexto organizacional, gerando fatores que auxiliam ou dificultam os fluxos desse processo. Esta pesquisa objetiva analisar os principais fatores facilitadores e obstáculos para abordagens direcionadas à gestão de riscos empresariais no setor da construção civil nacional. Para isso, utilizou como referência uma pesquisa de Cingapura, adaptando seu questionário e o aplicando em uma amostra não probabilística, delimitada por 102 profissionais e especialistas que atuam no setor da construção nacional. Os dados obtidos foram analisados estatisticamente, por meio do teste $\mathrm{t}$ de Student para amostra única, identificando 6 fatores facilitadores e 16 obstáculos, considerados críticos aos processos de gerenciamento de riscos em empresas de construção. Em seguida, os 22 fatores foram avaliados com base nas teorias de economia (do E) e de organização (do O), e comparados a fatores de resistência organizacional destacados na literatura. Os resultados mostraram que os itens relacionados a questões de burocrcia empresarial no cenário nacional apresentaram diferenças estatisticamente significantes, quando comparados aos processos empresariais de Cingapura.

Palavras-chave: Construção civil; Gestão do risco empresarial; Facilitadores de implementação; Obstáculos de implementação; Mudanças organizacionais.

\footnotetext{
Abstract

The use of a risk management system is one of the essential aspects for the survival of companies in the construction sector, especially in post-crisis times. However, the process of implementing this system requires changes in the organizational context, generating factors that help or hinder the flow of this process. This research aims to analyze the main facilitating factors and obstacles to approaches directed to business risk management in the national construction sector. To this end, it used a Singapore survey as a reference, adapting its questionnaire and applying it to a non-probabilistic sample, delimited by 102 professionals and specialists working in the national construction sector. The data obtained were analyzed statistically, using the Student's $t$ test for a single sample, identifying 6 facilitating factors and 16 obstacles, considered critical to the risk management processes in construction companies. Then, the 22 factors were evaluated based on the theories of economics (of E) and organization (of $\mathrm{O}$ ) and compared to factors of organizational resistance highlighted in the literature. The results showed that the items related to issues of business bureaucracy in the national scenario showed statistically significant differences when compared to the business processes in Singapore.
} 
Keywords: Civil construction; Business risk management; Implementation facilitators; Implementation obstacles; Organizational changes.

\begin{abstract}
Resumen
El uso de un sistema de gestión de riesgos es uno de los aspectos esenciales para la supervivencia de las empresas del sector de la construcción, especialmente en tiempos poscrisis. Sin embargo, el proceso de implementación de este sistema requiere cambios en el contexto organizacional, generando factores que ayuden u obstaculicen el flujo de este proceso. Esta investigación tiene como objetivo analizar los principales factores facilitadores y obstáculos de los enfoques dirigidos a la gestión del riesgo empresarial en el sector de la construcción nacional. Para ello, tomó como referencia una encuesta de Singapur, adaptando su cuestionario y aplicándolo a una muestra no probabilística, delimitada por 102 profesionales y especialistas que trabajan en el sector de la construcción nacional. Los datos obtenidos fueron analizados estadísticamente, utilizando la prueba t de Student para una sola muestra, identificando 6 factores facilitadores y 16 obstáculos, considerados críticos para los procesos de gestión de riesgos en las empresas constructoras. Luego, se evaluaron los 22 factores con base en las teorías de la economía (de E) y organización (de O), y se compararon con factores de resistencia organizacional resaltados en la literatura. Los resultados mostraron que los ítems relacionados con temas de burocracia empresarial en el escenario nacional mostraron diferencias estadísticamente significativas al compararlos con los procesos de negocio en Singapur.

Palabras clave: Construcción civil; Gestión de riesgos comerciales; Facilitadores de implementación; Obstáculos de implementación; Cambios en la organización.
\end{abstract}

\title{
1. Introdução
}

Após recessão econômica de 2015 a 2017, muitas empresas brasileiras do setor da construção civil passaram dificuldades para retomar seus níveis de investimento, reestabelecendo o crescimento do setor, que ainda sente os impactos da crise. Nesse cenário, temos o estado do Rio de Janeiro como um dos mais impactados com a falta de investimentos no setor.

Considerando que a indústria da construção civil exerce influência direta sobre diversos setores da economia nacional, com vários postos de trabalho, empregando, direta e indiretamente, 7,6\% da população ativa economicamente. Antes da crise, em 2014, apresentava um elevado potencial de investimento, com cerca de $\mathrm{R} \$ 307$ bilhões de valor bruto adicionado (IBGE, 2016).

Atualmente, a instabilidade econômica e a baixa demanda por obras representam consideráveis obstáculos para a sobrevivência das construtoras, que precisam controlar seus níveis de risco para se manterem no mercado. Nesse sentido, a implantação da gestão de riscos empresariais (GRE) surge como uma ferramenta direcionada pela estratégia das organizações para identificar fatores indesejáveis que possam impactar seus processos e monitorar os riscos (Ching, 2011).

Apesar da sua relevância para a estratégia das organizações, pode-se observar baixos níveis de aplicação de práticas de gerenciamento de risco (PGR) em diferentes partes do mundo. Como exemplo, temos grande parte das construtoras chinesas, que apresentam baixo percentual de aplicação das PGR, motivadas por elevada quantidade de obstáculos para suas implementações (Zhao, Hwang \& Low, 2014).

Cumpre destacar que literatura apresenta uma grande quantidade de estudos focados na implementação de gerenciamento de riscos empresariais em diversos segmentos e setores, ainda é pequena a quantidade de material que aborda os status de implementação no setor de construção, bem como dos fatores que facilitam ou dificultam as mudanças adaptadas para seus ambientes (Zhao, Hwang \& Low, 2014; Freitas, 2018).

Diante do exposto, esta pesquisa tem como objetivo, identificar os principais fatores, facilitadores e dificultadores, que afetam a implementação da gestão dos riscos em empresas da construção civil nacional. Para isso, com base no trabalho de Zhao, Hwang e Low (2014), desenvolveu-se uma survey para ser aplicada a profissionais da construção civil (engenheiros e arquitetos) atuantes em empresas do cenário nacional.

Em seguida, por meio da análise estatística dos dados, os fatores identificados são avaliados, com base nas teorias de economia (do E) e de organização (do O), e comparados aos fatores de resistência a mudanças organizacionais destacados na literatura. Por fim, os resultados da pesquisa são comparados à pesquisa de base, verificando se há diferenças estatisticamente 
significativas entre os aspectos nacionais e os de Cingapura, fonte de base para esta pesquisa.

\section{Revisão da literatura}

\subsection{Mudança organizacional}

A mudança organizacional consiste em uma observação empírica na diferença de formas, qualidade ou estado de uma entidade organizacional ao longo prazo, que tem sua fundamentação na introdução deliberada de novos estilos de pensamento, ações ou operações, que buscam adaptar o ambiente à melhoria de desempenho para fortalecimento, desenvolvimento e sobrevivência das organizações presentes em ambientes voláteis. Com base nesse entendimento, Zhao, Low e Hwang (2014) destacam e identificam vinte e uma fontes de resistência à mudança organizacional, conforme mostra o Quadro 1.

Quadro 1: Forças internas e externas de mudança organizacional.

\begin{tabular}{|c|l|}
\hline Código $(\mathbf{R})$ & \\
\hline 01 & Hábitos \\
\hline 02 & Medo do desconhecido \\
\hline 03 & Interesse próprio paroquial \\
\hline 04 & Fatores sociais \\
\hline 05 & Falta de capacidade individual para mudar à mudança organizacional \\
\hline 06 & Mal-entendido \\
\hline 07 & Recursos insuficientes \\
\hline 08 & Recompensas e punições inadequadas \\
\hline 09 & Má comunicação interna \\
\hline 10 & Falta de comprometimento do conselho e da alta administração \\
\hline 11 & Falta de confiança na gestão \\
\hline 12 & Inconsistências \\
\hline 13 & Baixo nível de relação empregado-gerente \\
\hline 14 & Estilos de gerenciamento ineficazes \\
\hline 15 & Processamento seletivo de informações \\
\hline 16 & Ameaças ao poder ou influência \\
\hline 17 & Ameaças à alocação de recursos \\
\hline 18 & Foco limitado de mudança \\
\hline 19 & Cultura organizacional \\
\hline 20 & Inércia do grupo \\
\hline 21 & Inércia estrutural \\
\hline
\end{tabular}

Fonte: Adaptado de Zhao, Low e Hwang (2014).

Importante destacar que, nos fatores de resistência às mudanças presentes no Quadro 1, os aspectos de recursos insuficientes (R07) podem ser diretamente relacionados com problemas econômicos. Assim como, os problemas de cultura organizacional e de inércia do grupo (R19 e R20) têm associação direta com fatores de origem organizacional (Beer \& Noria, 2000).

Sob uma ótica mais ampla, Bressian (2004) destaca que a mudança organizacional é inicialmente caracterizada por fatores associados a resistência. Dessa forma, qualquer forma de mudança, independentemente de seu tipo ou objetivo, não tende a ser facilmente aceita. Assim, a resistência à mudança configura uma resposta natural dos indivíduos, com base no pressuposto de que seus comportamentos anteriores sempre funcionaram de forma adequadamente satisfatória para as organizações (Zhao, Low \& Hwang, 2014).

Além dos aspectos relacionados à resistência, Bressan (2004) e Zhao, Low e Hwang (2014) consideram que a mudança organizacional é provocada pelo surgimento de forças internas e externas que caracterizam sua necessidade de implantação, conforme mostra o Quadro 2. 
Quadro 2: Forças internas e externas de mudança organizacional.

\begin{tabular}{|l|l|}
\hline \multicolumn{1}{|c|}{ Forças internas (FI) } & \multicolumn{1}{c|}{ Forças externas (FE) } \\
\hline 1 Necessidade de reorganização; & 1 Avanços técnicos; \\
2 Necessidade de maior lucratividade; & 2 Globalização; \\
3 Conflito entre componentes organizacionais; & 3 Pressões da concorrência; \\
4 Natureza mutável e a composição da força de trabalho. & 4 Fatores sociais e culturais; \\
5 Desempenho; & 5 Fatores econômicos; \\
6 Características da gestão; & 6 Pressões políticas e jurídicas; \\
7 Natureza da força do trabalho; & 7 Mudanças de mercado; \\
8 Crescimento organizacional; & 8 Política mundial; \\
9 Descontinuidade na estrutura organizacional & 9 Crises e tendências macroeconômicas; \\
& 10 Mudanças legais e regulamentares \\
& 11 Recessão econômica; \\
& 12 Competição e inovação tecnológica. \\
\hline
\end{tabular}

Fonte: Adaptado de Zhao, Low e Hwang (2014), e Bressan (2004).

Observa-se que as forças internas e externas apontadas em Quadro 2 também apresentam relação com motivações econômicas e organizacionais. As forças internas FI 01, FI 02, FI 03, FI 06, FI 08, FI 09 estão relacionadas a problemas organizacionais, enquanto a força interna FI 02 e as forças externas FE 03, FE 05, FE 09, FE 11 estão relacionados a problemas econômicos.

\subsection{Teorias econômica e organizacional}

De acordo com Beer e Noria (2000), 70\% dos programas de implantação de mudança em empresas falham. Muito dessas falhas podem ser associadas a excessos de métodos que podem ser conflitantes e apresentarem elevados níveis de esforços econômicos e culturais. Por esse motivo, sugere-se que as organizações abordem duas teorias elementares de mudança organizacional: a teoria econômica, também conhecida como teoria " $d o$ E"; e a teoria organizacional, também conhecida como teoria "do $O$ ".

Como definição básica, a teoria "do E" enfatiza os resultados econômicos, por meio de ações que visam um retorno financeiro no curto prazo, como demissões e reestruturações que priorizam a posição dos acionistas. Já a teoria “do $O$ " segue por uma abordagem menos agressiva, tendo seu foco no desenvolvimento da cultura corporativa e da capacidade do capital humana, com base em um relacionamento sólido de confiança e comprometimento com os valores da empresa, por meio do trabalho em equipe e da comunicação eficiente (Beer \& Noria, 2000).

\subsection{Implementação do gerenciamento de riscos na construção}

As atividades inerentes ao ser humano, desde os primórdios, estão intrinsecamente ligadas a algum potencial de risco. No setor da construção, assim como no mundo dos negócios, todas as definições e ações das empresas envolvem algum tipo de risco que resulta em impactos - positivos ou negativos. Os efeitos provenientes desses impactos podem resultar em diversos fatores indesejados (Ruppenthal, 2013; Renault \& Agumba, 2016).

A implementação do gerenciamento de riscos contempla a definição das práticas de planejamento, avaliação de desempenho e análise críticas dos resultados. A etapa de avaliação do processo de implementação possibilita uma melhor visão do status quo às empresas, permitindo que a equipe de gerenciamento conduza inicialmente, ações de melhoria direcionadas às áreas com maiores capacidades de fragilidade (Zhao, Low \& Hwang, 2014).

A etapa inicial da construção de um processo funcional de gestão de riscos consiste na identificação do nível de 
implementação que a empresa se encontra e quais devem ser os requisitos necessários para que a organização possa atingir um potencial adequado de desempenho para seu sistema de gerenciamento de riscos. No entanto, muitas empresas enxergam o gerenciamento de riscos como ferramenta de gestão, ao invés de uma ferramenta de boas práticas. Esse aspecto resulta em níveis insuficientes de maturidade na condução dessa ferramenta, o que induz a problemas relacionados à complexidade dos processos de mudança organizacional (Ito, 2018).

De acordo com Renault e Agumba (2016), o desafio de gerenciar os riscos presentes em projetos de construção é uma atividade altamente complexa, que é potencializada em função das variáveis presentes nos ambientes interno e externo. Dentre as variáveis existentes, podem ser destacadas: a dimensão do projeto, níveis de concorrência, exigências do cliente, desafios político-econômicos e limitações físicas.

Entre os fatores facilitadores identificados da literatura, 17 deles foram encontrados em trabalhos publicados sobre a atuação de empresas chinesas no ambiente asiático, que estão relacionados à redução da volatilidade de ganhos, redução de custos e prejuízos, melhoramento nas tomadas de decisão e na obtenção de vantagens competitivas. Em complemento, foram identificados 5 fatores facilitadores associados com a ISO 31000 (2009). O Quadro 3 apresenta os 22 fatores facilitadores encontrados na literatura.

Quadro 3: Facilitadores para implementação do sistema de gerenciamento dos riscos.

\begin{tabular}{|c|c|c|}
\hline Itens & Fatores facilitadores & Referências \\
\hline 1 & Requisitos legais e de conformidade regulamentar & \multirow{8}{*}{$\begin{array}{c}\text { Zhao, Hwang \& Low, } \\
\text { 2014a. } \\
\text { Zhao, Hwang \& Low, } \\
\text { 2014b. }\end{array}$} \\
\hline 2 & Não obrigatoriedade de relatórios ou padrões & \\
\hline 3 & Requisitos das agências de notação de crédito & \\
\hline 4 & A redução da volatilidade das captações & \\
\hline 5 & A redução de custos e perdas & \\
\hline 6 & O aumento da rentabilidade e dos ganhos & \\
\hline 7 & Melhorias na tomada de decisões & \\
\hline 8 & Melhorias do relato de risco e na comunicação & \\
\hline 9 & O aumento da responsabilidade gerencial & \multirow{3}{*}{$\begin{array}{c}\text { Zhao, Hwang, Low \& } \\
\text { Wu, } 2015 .\end{array}$} \\
\hline 10 & $\mathrm{O}$ aumento do consenso de gestão & \\
\hline 11 & Busca por incessante por vantagens competitivas & \\
\hline 12 & Melhoria na alocação dos recursos & \multirow{6}{*}{$\begin{array}{c}\text { Zhao \& } \\
\text { Singhaputtangkul, } \\
2016 .\end{array}$} \\
\hline 13 & Melhoria na satisfação dos proprietários da empresa & \\
\hline 14 & Melhoria no controle dos projetos & \\
\hline 15 & Âmbito de riscos no estrangeiro & \\
\hline 16 & Avanços em tecnologia de TI & \\
\hline 17 & Incentivos da alta gestão & \\
\hline 18 & Aumento na probabilidade de atingimento das metas & \multirow{5}{*}{ ISO 31000 (2009) } \\
\hline 19 & Melhoria na identificação de oportunidades e ameaças & \\
\hline 20 & Melhoria nos registros financeiros & \\
\hline 21 & Melhoria nos níveis de confiança dos Stakeholders & \\
\hline 22 & Melhoria do aprendizado organizacional. & \\
\hline
\end{tabular}

Fonte: Autores.

Com relação aos obstáculos para a implementação do gerenciamento de riscos em empresas de construção, foram identificados na literatura, 50 fatores complicadores, em diversas pesquisas que abordam, principalmente questões relacionadas a dados, recursos e fatores organizacionais, associados com a estrutura do sistema de gerenciamento de riscos, conforme mostra o Quadro 4. 
Quadro 4: Fatores obstáculos para implementação do sistema de gerenciamento de riscos.

\begin{tabular}{|c|c|c|}
\hline Item & Fator obstáculo para implementação & Referências \\
\hline 1 & Baixa qualidade de dados & \multirow{50}{*}{$\begin{array}{c}\text { Zhao, Hwang \& } \\
\text { Low, 2014a. } \\
\\
\text { Zhao, Hwang \& } \\
\text { Low, 2014b. } \\
\text { Zhao, Hwang, } \\
\text { Low \& Wu, 2015. } \\
\text { Zhao \& } \\
\text { Singhaputtangkul, } \\
2016\end{array}$} \\
\hline 2 & Ausência de dados & \\
\hline 3 & Recursos insuficientes & \\
\hline 4 & Falta de formalização do processo de gerenciamento de riscos & \\
\hline 5 & Falta de técnicas e ferramentas de gerenciamento de riscos & \\
\hline 6 & Falta de competências internas & \\
\hline 7 & Falta de pessoal qualificado para implementar o sistema de gerenciamento de riscos & \\
\hline 8 & Falta de um sistema de informações de gerenciamento de riscos & \\
\hline 9 & Estrutura organizacional não-suportada & \\
\hline 10 & Cultura organizacional não apoiadora & \\
\hline 11 & Falta de uma linguagem padronizada de riscos & \\
\hline 12 & Falta de consciência dos riscos dentro da organização & \\
\hline 13 & Confiança nas práticas existentes de gerenciamento de riscos & \\
\hline 14 & Mentalidade do silo & \\
\hline 15 & Falta de compreensão e compartilhamento do gerenciamento de riscos nos departamentos & \\
\hline 16 & Falta de entendimento relacionado aos processos efetivos do gerenciamento de riscos & \\
\hline 17 & Percepção de que o sistema de gerenciamento de riscos aumenta a burocracia & \\
\hline 18 & Percepção de que o sistema de gerenciamento de riscos aumenta custos de administração & \\
\hline 19 & Percepção de que o sistema de gerenciamento de riscos interfere nas atividades de negócios & \\
\hline 20 & Treinamento inadequado do sistema de gerenciamento de riscos & \\
\hline 21 & Falta de um case de negócio como referência do sistema de gerenciamento de riscos & \\
\hline 22 & Falta de valor percebido ou benefícios do sistema de gerenciamento de riscos & \\
\hline 23 & Falta de comprometimento do conselho e da alta gestão & \\
\hline 24 & Falta de percepção de prioridade para o sistema de gerenciamento de riscos & \\
\hline 25 & Falta de diretoria ou liderança de gerência sênior para o sistema de gerenciamento de riscos & \\
\hline 26 & O movimento do campeão da GRE da alta administração para outras áreas sem um sucessor & \\
\hline 27 & $\begin{array}{l}\text { Falta de consenso sobre os benefícios do sistema de gerenciamento de riscos entre os membros do } \\
\text { conselho e a alta administração }\end{array}$ & \\
\hline 28 & Diversas prioridades da gestão & \\
\hline 29 & Falta de um plano claro de implementação do sistema de gerenciamento de riscos & \\
\hline 30 & Falta de capacidade de coordenação com outros departamentos & \\
\hline 31 & Falta de um conjunto de métricas para medir o desempenho do sistema de gerenciamento de riscos & \\
\hline 32 & Propriedade e responsabilidade obscuras para a implementação do sistema de gerenciamento de riscos & \\
\hline 33 & Relógio Organizacional & \\
\hline 34 & A relutância dos funcionários em desistir do poder & \\
\hline 35 & Relutância dos funcionários em compartilhar informações de risco & \\
\hline 36 & Recessão nos negócios & \\
\hline 37 & Dificuldade para identificar o risco e suas fontes & \\
\hline 38 & Dificuldades para definir e implementar resposta aos riscos & \\
\hline 39 & Dificuldades para entender a organização e seu contexto & \\
\hline 40 & Falta de relacionamento com a alta direção & \\
\hline 41 & Dificuldade de monitoramento das respostas da implementação & \\
\hline 42 & Dificuldade para estruturar setores do risco & \\
\hline 43 & Dificuldade para identificar alterações no ambiente & \\
\hline 44 & Dificuldades para estabelecer objetivos, estratégia e parâmetros para o escopo & \\
\hline 45 & Dificuldade para definir critérios e limites de aceitação do risco & \\
\hline 46 & Dificuldades para definir o planejamento operacional para o sistema de gerenciamento de riscos & \\
\hline 47 & Dificuldades para identificar consequências de ações & \\
\hline 48 & Dificuldade em desenvolver resposta às contingências & \\
\hline 49 & Dificuldade em priorizar os resultados de acordo com os critérios prévios & \\
\hline 50 & Dificuldade em tratar o risco residual & \\
\hline
\end{tabular}

Fonte: Autores.

Além da elevada quantidade e complexidade de variáveis, e todos os desafios relacionados à construção, deve-se 
considerar nesse escopo a presença dos fatores facilitadores e complicadores no processo de implementação do sistema de gerenciamento de riscos em empresas de construção.

\section{Metodologia}

Esta pesquisa tem por finalidade identificar os principais fatores, facilitadores e dificultadores, que afetam a implementação do sistema de gerenciamento dos riscos em empresas da construção civil nacional.

A característica de abordagem é quantitativa, com finalidade exploratória, fundamentada pela aplicação de survey e procedimentos estatísticos. Para efeito de estudo, os dados obtidos pela survey foram analisados estatisticamente e submetidos ao teste $\mathrm{t}$ de student para amostra única, onde os resultados obtidos pelo grupo são comparados a um escore previamente existente.

Os critérios adotados podem ser traduzidos, de forma prática, por meio da coleta e da análise de dados. Tais recursos requerem conhecimento específico do pesquisador para a abordagem adequada dos dados numéricos obtidos (Creswell, 2012).

\subsection{Caracterização da amostra}

Considerando que a amostra do estudo foi escolhida para representar uma população da qual pertence e que, devido ao não conhecimento da real dimensão de quantos seriam os profissionais com experiência em projetos de construção e conhecimento dos riscos presentes no ambiente, seria custoso, demorado ou inviável estudar sua totalidade. Por esse motivo, optou-se por uma amostragem não-probabilística por conveniência, caracterizada por profissionais de engenharia e arquitetura, com conhecimentos específicos sobre o tema, em sua grande maioria localizados no estado do Rio de Janeiro.

A utilização da amostragem por conveniência se justifica por ser um dos tipos de seleção de amostra mais usualmente aplicados em estudos, cujos aspectos geográficos podem ser superados pela disponibilidade do pesquisador, que busca maior facilidade para a obtenção dos dados a serem coletados (Farrokhi \& Mahmoudi-hamidabad, 2012; Freitas, 2018).

Com relação ao tamanho da amostra, Leech (2005) destaca que, por apresentarem valores muito próximos, quanto maior a quantidade de elementos observados (n), melhores serão os resultados obtidos. Considera grande o valor de n, quando $n>50$ e $(n-p)>50$. Neste caso, p é a quantidade das variáveis que serão analisadas. No entanto, deve-se ter cautela, quando $(n-p)<50$.

Com base nesse entendimento, esta pesquisa adotou como condição mais restritiva a abordagem dos fatores obstáculos, pois contemplam maior número de variáveis $(p=50)$, ou seja, esse questionário foi composto por 50 variáveis e respondido por 102 profissionais atuantes no setor da construção civil. Sua configuração atende aos critérios definidos por Leech (2005).

\subsection{Definição e desenvolvimento do instrumento de pesquisa}

A elaboração do questionário da pesquisa foi fundamentada a partir da análise das referências bibliográficas, que identificou 22 fatores facilitadores e 50 fatores complicadores, ou obstáculos, para implementação de sistemas de gerenciamento de riscos em empresas da construção civil, conforme mostram os Quadros 3 e 4.

A partir da identificação dos fatores, foram elaboradas questões assertivas relacionadas a cada fator facilitador e complicador. Um questionário estruturado foi desenvolvido, tendo como critério a utilização de uma escala tipo Likert, pontuada de 1 a 5 , onde os valores equivalem a: 1 - nada importante; 2 - pouco importante; 3 - mais ou menos importante; 4 importante; e 5 - extremamente importante.

A plataforma utilizada para aplicação da pesquisa foi o google forms, o que facilitou a coleta dos dados. Além das questões relacionadas aos fatores facilitadores e complicadores para a implementação do sistema de gerenciamento de riscos, verificou-se se perfil a adequação dos respondentes à amostragem definida, onde destacam-se as informações relativas a: 
formação acadêmica, tempo de experiência na profissão, nível de conhecimento do tema.

\subsection{Análise estatística dos dados}

A literatura apresenta diversos tipos de testes para verificar a significância dos dados, que podem ser realizados, conforme suas características (se contados ou medidos), da quantidade de amostras e do propósito da medição. Para isso, o tipo mais utilizado de avaliação é o teste t, cuja origem vem da distribuição t de Student, comumente empregada para verificar a aproximação da média amostral.

O teste t é uma das técnicas estatísticas mais populares usadas para testar se a diferença média entre dois grupos é estatisticamente significativa. A hipótese nula afirmava que ambos os meios são estatisticamente iguais, enquanto a hipótese alternativa afirmava que ambas as médias não são estatisticamente iguais, ou seja, são estatisticamente diferentes entre si (Sundaram, Dwivedi \& Sreenivas, 2014). O teste t é caracterizado por três tipos de aplicação: de amostra única, de amostras independentes e de amostras pareadas.

Teste $\mathrm{t}$ de amostra única, usado por esta pesquisa, é aplicado para determinar se o valor médio de uma amostra está estatisticamente semelhante ou diferente do valor médio de sua população, da qual a amostra foi retirada. Para executar este teste, deve-se considerar o desvio padrão amostral, tamanho da amostra e média da população ou valor médio hipotético. A amostra deve ser caracterizada por variáveis contínuas normalmente distribuídas (Sundaram, Dwivedi \& Sreenivas, 2014).

\section{Resultados}

\subsection{Fatores críticos para implementação da gestão de risco empresarial}

A técnica do teste t para amostra única foi realizada com o objetivo de verificar se os fatores apresentam significância estatística para os aspectos relacionados à implementação da Gestão de Risco Empresarial, conforme mostra a Tabela 1.

Tabela 1: Resultados da aplicação do teste $t$ de amostra única.

\begin{tabular}{|c|c|c|c|c|c|c|c|c|}
\hline \multicolumn{3}{|c|}{ Facilitadores } & & \multicolumn{5}{|c|}{ Obstáculos } \\
\hline Item & Média & $p$ & Item & Média & $p$ & Item & Média & $p$ \\
\hline F01 & 4,46 & $0,000^{*}$ & O01 & 4,41 & $0,000^{*}$ & O26 & 3,99 & 0,909 \\
\hline F02 & 3,54 & 0,000 & $\mathrm{O} 02$ & 4,54 & $0,000 *$ & $\mathrm{O} 27$ & 3,97 & 0,741 \\
\hline F03 & 3,91 & 0,307 & $\mathrm{O} 03$ & 4,19 & $0,041 *$ & $\mathrm{O} 28$ & 3,62 & 0,000 \\
\hline F04 & 3,82 & 0,069 & $\mathrm{O} 04$ & 4,13 & 0,139 & O29 & 4,15 & 0,075 \\
\hline F05 & 3,92 & 0,435 & O05 & 4,29 & $0,000 *$ & $\mathrm{O} 30$ & 4,05 & 0,525 \\
\hline F06 & 3,77 & 0,023 & O06 & 4,21 & $0,017^{*}$ & O31 & 4,05 & 0,581 \\
\hline F07 & 4,47 & $0,000 *$ & O07 & 4,31 & $0,000 *$ & $\mathrm{O} 32$ & 4,04 & 0,620 \\
\hline F08 & 4,63 & $0,000 *$ & O08 & 4,10 & 0,220 & $\mathrm{O} 33$ & 3,61 & 0,000 \\
\hline F09 & 4,13 & 0,139 & O09 & 4,31 & $0,000 *$ & $\mathrm{O} 34$ & 3,69 & 0,001 \\
\hline F10 & 4,04 & 0,644 & $\mathrm{O} 10$ & 4,16 & 0,059 & O35 & 4,12 & 0,146 \\
\hline F11 & 3,59 & 0,000 & O11 & 4,00 & 1,000 & O36 & 3,97 & 0,777 \\
\hline F12 & 3,99 & 0,908 & $\mathrm{O} 12$ & 4,32 & $0,000^{*}$ & O37 & 4,25 & $0,002 *$ \\
\hline F13 & 3,63 & 0,001 & O13 & 3,78 & 0,044 & O38 & 4,16 & 0,099 \\
\hline F14 & 4,35 & $0,000 *$ & $\mathrm{O} 14$ & 3,78 & 0,030 & O39 & 3,99 & 0,905 \\
\hline F15 & 4,13 & 0,160 & O15 & 4,00 & 1,000 & O40 & 4,05 & 0,590 \\
\hline F16 & 4,00 & 1,000 & O16 & 4,12 & 0,140 & O41 & 3,74 & 0,025 \\
\hline F17 & 4,19 & $0,021 *$ & O17 & 3,75 & 0,007 & O42 & 3,94 & 0,488 \\
\hline F18 & 3,91 & 0,363 & $\mathrm{O} 18$ & 3,87 & 0,206 & O43 & 3,91 & 0,294 \\
\hline F19 & 4,15 & 0,128 & O19 & 3,73 & 0,004 & O44 & 4,29 & $0,001 *$ \\
\hline $\mathrm{F} 20$ & 3,77 & 0,041 & $\mathrm{O} 20$ & 4,25 & $0,004 *$ & O45 & 4,30 & $0,000 *$ \\
\hline F21 & 4,02 & 0,820 & $\mathrm{O} 21$ & 3,87 & 0,160 & O46 & 4,26 & $0,003 *$ \\
\hline $\mathrm{F} 22$ & 4,21 & $0,024 *$ & $\mathrm{O} 22$ & 3,87 & 0,164 & O47 & 4,17 & 0,059 \\
\hline- & - & - & $\mathrm{O} 23$ & 4,46 & $0,000 *$ & O48 & 4,02 & 0,836 \\
\hline- & - & - & $\mathrm{O} 24$ & 4,40 & $0,000 *$ & O49 & 3,91 & 0,326 \\
\hline- & - & - & $\mathrm{O} 25$ & 4,32 & $0,000 *$ & O50 & 3,79 & 0,032 \\
\hline
\end{tabular}


Fonte: Autores.

De acordo com os pressupostos da análise do teste t de Student, por meio dos testes de Kolmogorov-Smirnov (K-S) e Shapiro-Wilk (S-W), verificou-se se a distribuição dos dados presentes nos fatores facilitadores e obstáculos apresentavam distribuição normal, conforme mostra a Tabela 2.

Tabela 2: Aplicação dos testes K-S e S-W.

\begin{tabular}{lcccccc} 
& \multicolumn{3}{c}{ Kolmogorov-Smirnov } & \multicolumn{3}{c}{ Shapiro-Wilk } \\
\hline Fatores & Estatística & df & Sig. & Estatística & df & Sig. \\
\hline Facilitadores & 0,083 & 22 &, $200^{*}$ & 0,977 & 22 & 0,872 \\
Obstáculos & 0,073 & 50 &, $200^{*}$ & 0,983 & 50 & 0,661 \\
\hline
\end{tabular}

Correlação de Significância de Lilliefors

Fonte: Autores.

Importante destacar que nos testes de Kolmogorov-Smirnov (K-S) e Shapiro-Wilk $(\mathrm{S}-\mathrm{W})$, espera-se que $(p>0,05)(p$ maior do que 0,05 ) para acatar a distribuição da normalidade dos dados.

Em seguida, com base na análise estatística da amostra, identificou-se os valores da média de cada grupo, bem como seus respectivos erros (desvio e padrão da média), conforme mostra a Tabela 3.

Tabela 3: Análise da estatística da amostra.

\begin{tabular}{ccccc}
\hline Fatores & N & Média & Desvio padrão & Erro padrão da média \\
\hline Facilitadores & 22 & 4,029 & 0,289 & 0,062 \\
Obstáculos & 50 & 4,064 & 0,227 & 0,032 \\
\hline
\end{tabular}

Fonte: Autores.

Por fim, considerando o valor arbitrado para a execução da análise $(t=4)$, foram aplicadas as análises do teste de $\mathrm{t}$ student para verificar a significância estatística da amostra aos fatores de facilidade e obstáculos associados ao modelo adotado.

A Tabela 4 apresenta os resultados obtidos.

Tabela 4:

\begin{tabular}{|c|c|c|c|c|c|c|}
\hline \multirow[t]{2}{*}{ Fatores } & \multirow[t]{2}{*}{$\mathbf{t}$} & \multirow[t]{2}{*}{ df } & \multirow{2}{*}{$\begin{array}{c}\text { Sig. } \\
(2 \text { extremidades })\end{array}$} & \multirow{2}{*}{$\begin{array}{l}\text { Diferença } \\
\text { média }\end{array}$} & \multicolumn{2}{|c|}{$\begin{array}{l}\text { 95\% Intervalo de Confiança da } \\
\text { Diferença }\end{array}$} \\
\hline & & & & & Inferior & Superior \\
\hline Facilitadores & 0,465 & 21 & 0,647 & 0,02864 & $-0,0993$ & 0,1566 \\
\hline Obstáculos & 1,998 & 49 & 0,051 & 0,06420 & $-0,0004$ & 0,1288 \\
\hline
\end{tabular}

Fonte: Autores.

A aplicação do teste $\mathrm{t}$ de Student para amostra única objetivou investigar em que medida o nível dos fatores facilitadores e obstáculos se apresentava inferior ao valor estipulado $(M=4)$.

Os resultados demonstraram que, não houve diferença estatisticamente significativa entre a média da amostra $(M=$ 4,029; $D P=0,289)$ e a média esperada $(t(21)=0,465 ; p=0,647)$ nos fatores facilitadores, bem como nos fatores obstáculos, 
que apresentaram $(M=4,064 ; D P=0,227)$ e $(t(49)=1,998 ; p=0,051)$. Os resultados da análise indicam que 6 facilitadores e 16 obstáculos apresentam escores médios acima de 4,00 e $p<0,05$, conforme mostra a Tabela 5.

Tabela 5: Fatores críticos obtidos da aplicação do teste t de amostra única.

\begin{tabular}{ccc|ccc|ccc}
\hline & Facilitadores & & \multicolumn{5}{c}{ Obstáculos } \\
\hline Item & Média & $\mathbf{p}$ & Item & Média & $\mathbf{p}$ & Item & Média & p \\
\hline F01 & 4,46 & $0,000^{*}$ & O01 & 4,41 & $0,000^{*}$ & O20 & 4,25 & $0,004^{*}$ \\
F07 & 4,47 & $0,000^{*}$ & O02 & 4,54 & $0,000^{*}$ & O23 & 4,46 & $0,000^{*}$ \\
F08 & 4,63 & $0,000^{*}$ & O03 & 4,19 & $0,041^{*}$ & O24 & 4,4 & $0,000^{*}$ \\
F14 & 4,35 & $0,000^{*}$ & O05 & 4,29 & $0,000^{*}$ & O25 & 4,32 & $0,000^{*}$ \\
F17 & 4,19 & $0,021^{*}$ & O06 & 4,21 & $0,017^{*}$ & O37 & 4,25 & $0,002^{*}$ \\
F22 & 4,21 & $0,024^{*}$ & O07 & 4,31 & $0,000^{*}$ & O44 & 4,29 & $0,001^{*}$ \\
- & - & - & O09 & 4,31 & $0,000^{*}$ & O45 & 4,3 & $0,000^{*}$ \\
- & - & - & O12 & 4,32 & $0,000^{*}$ & O46 & 4,26 & $0,003^{*}$ \\
\hline
\end{tabular}

Resultado de Teste t de uma amostra $(t=4,0 ; p<0,05)$

Fonte: Autores.

Assim como no artigo de Zhao, Low e Hwang (2014), o fator (F07) "melhor tomada de decisão" foi apontado como crítico para implementação, por motivar os CEO's das empresas de construção do mercado global. Por outro lado, o fator (F01) "Requisitos legais e de conformidade regulamentar" foi identificado como facilitador crítico somente no Brasil, o que pode ser explicado pelo excesso de legislações locais ou por questões regionais em que mudanças ocorrem em grande parte por necessidades legais.

Os complicadores (O01 e O02) "Baixa qualidade dos dados" e "Falta de dados" vão na mesma direção do facilitador (F08) "melhor relato do risco e comunicação" indicando que fonte de dados de risco junto a melhor compartilhamento desses dados são extremamente importantes nas organizações. O complicador (O03) "Recursos insuficientes (por exemplo, tempo, dinheiro, pessoas, etc.)" aparece como crítico assim como no estudo de Zhao, Low e Hwang (2014), que menciona que de forma semelhante ocorreu nos EUA, Europa e Dubai.

Como a implementação do GRE em empresas de construção pode ser considerada uma mudança organizacional, este estudo busca interpretar os itens da pesquisa e seus principais obstáculos na perspectiva da mudança organizacional, conforme mencionado anteriormente.

\subsection{Interpretação dos fatores facilitadores críticos sob perspectiva de mudanças organizacionais}

Zhao, Low e Hwang (2014) citam que a mudança organizacional pode ser alcançada através da Teoria E e da Teoria O. Novas metodologias podem ser relacionadas a investimentos, o que pode levar algumas organizações brasileiras a somente realizar mudanças estruturais que demandem investimento por necessidades legais, como novas leis ou novas regulamentações (F01), o que sugeriu a Teoria E poderia ser usada para implementar o GRE nas Empresas de Construção civil no Brasil.

Em contrapartida, "tomada de decisão aprimorada" (F07), "melhor comunicação e relatórios de risco" (F08), "Controle aprimorado de uma empresa sobre seus projetos" (F14), "Solicitação e incentivo do conselho e da diretoria executiva (17) e "melhora do aprendizado organizacional" (F22), representam o desenvolvimento da capacidade organizacional, indicando que a Teoria $\mathrm{O}$ também era aplicável à implementação do GRE. Portanto, as duas teorias podem ser usadas para implementar a Gestão de Risco Empresarial nas empresas de Construção civil no Brasil e substanciaram o argumento de Zhao, Low e Hwang (2014) de que a combinação de ambas as teorias foi a estratégia mais bem-sucedida para a mudança organizacional em Cingapura.

Além das teorias econômicas e organizacionais, os "requisitos legais e de conformidade regulamentar" (F01) constitui 
uma pressão política ou jurídica, abordado por Zhao, Low e Hwang (2014) como fonte de pressão externa na organização. Já a "tomada de decisão aprimorada" (F07), "melhor comunicação e relatórios de risco" (F08), "Controle aprimorado de uma empresa sobre seus projetos" (F14) são pressões internas relacionadas a necessidade de reorganização.

Os 17 obstáculos críticos foram interpretados em conjunto com algumas das 21 fontes de resistência à mudança organizacional (ver Tabela 7). Especificamente, 9 obstáculos significativos (O1 a O7, $\mathrm{O} 23$ a O25) podem representar "recursos insuficientes" (R07) nas fontes de resistência à mudança organizacional.

\subsection{Interpretação dos fatores obstáculos críticos sob ótica das mudanças organizacionais}

Conforme Zhao, Hwang e Low (2014) destacam, para promover a mudança organizacional de implementação do GRE é necessário ter uma variedade de recursos, incluindo tempo e pessoas, qualidade dos dados, conhecimento interno, habilidades e conhecimentos, técnicas, ferramentas e sistemas de informação para gerenciamento de riscos, além de métricas necessárias para avaliar o desempenho do GRE e cases de negócios para programas de treinamento.

Caso haja indisponibilidade ou insuficiência de recursos, pode ocorrer insatisfações ou frustrações junto à equipe de implementação, ocasionando picos de resistências ao longo da organização, provocando o desenvolvimento de um novo planejamento de alocação para os recursos, podendo resultar no aumentar do prazo previamente estipulado, conforme demonstrado na Figura 1.

Figura 1: Implementação de Gestão de risco empresarial em empresas de construção civil: sob perspectiva de mudanças organizacionais.

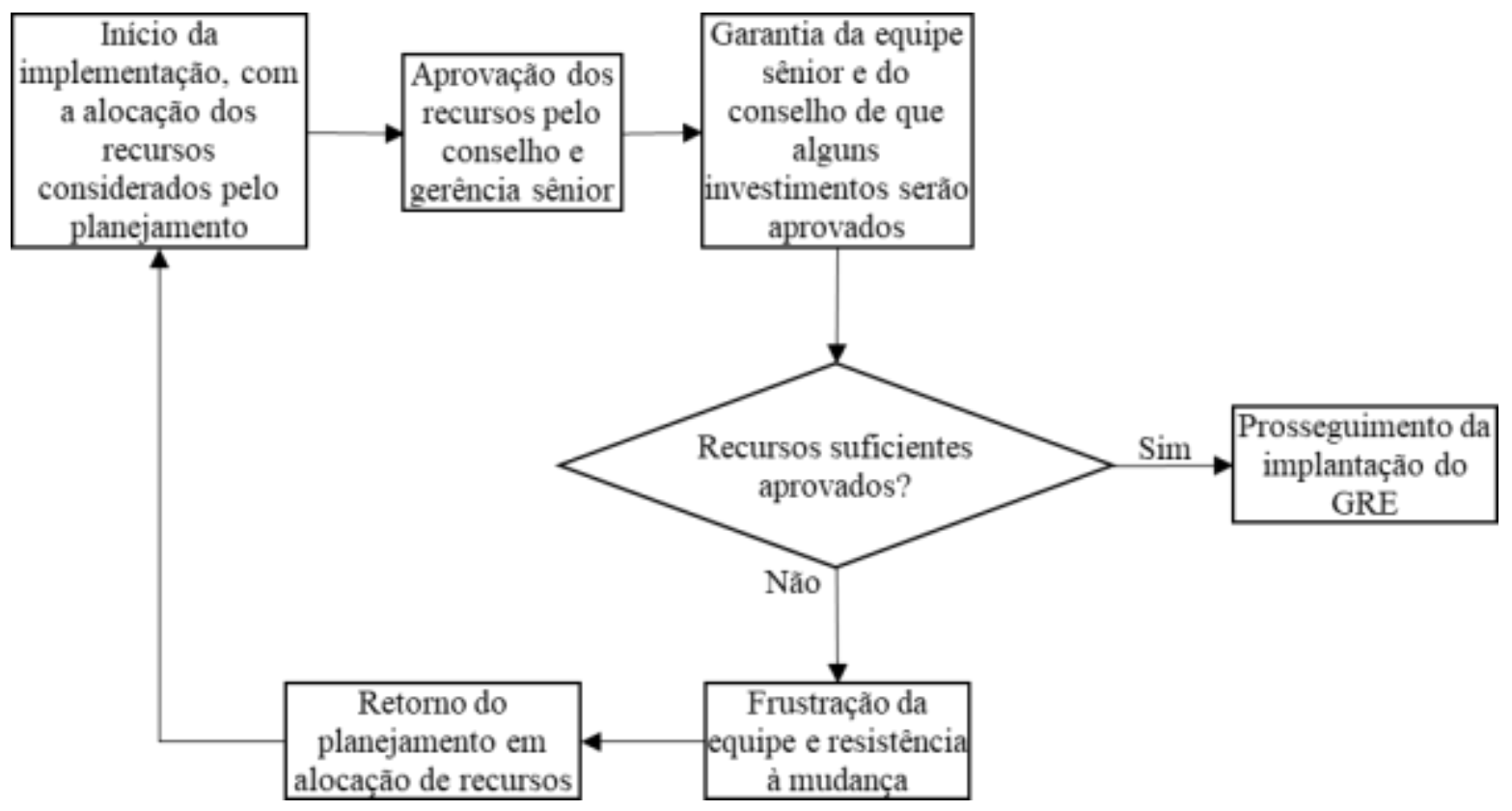

Fonte: Adaptado de Zhao, Hwang e Low (2014).

Além de da suficiência dos recursos, para implementar uma mudança organizacional, o agente de mudança deve garantir que as equipes de gerenciamento de risco possam se adaptar e participar do programa de mudanças, por esse motivo, a utilização de técnicas e ferramentas de gestão de risco (O05) é imprescindível ao processo. A ausência dessas técnicas e ferramentas fica associada ao item de resistência, configurado pela "falta de capacidade individual para mudar (R05).

Zhao, Low e Hwang (2014) consideram que a equipe passaria a questionar sua própria competência para a condução de uma implementação do GRE em termos de conhecimento, habilidades, experiência e capacidades. Se essa possibilidade 
existir, não terão condições de participar ativamente dos processos de implementação da GRE. A Tabela 6 apresenta os resultados das análises entre os fatores de resistência organizacional e os fatores associados aos obstáculos críticos abordados pelo modelo.

Tabela 6: Obstáculo crítico x Fator de Resistência Organizacional.

\begin{tabular}{|c|c|c|c|c|c|c|c|c|c|c|c|c|c|c|c|c|c|}
\hline 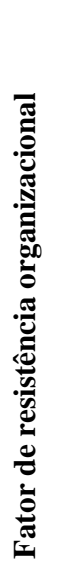 & 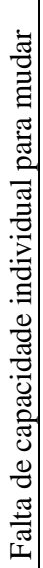 & 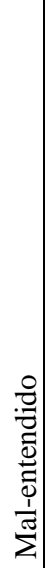 & 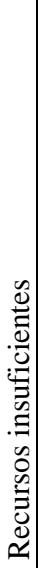 & 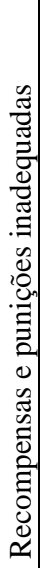 & 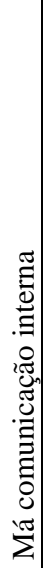 & 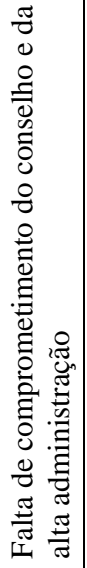 & 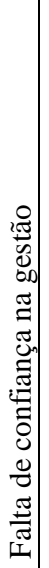 & 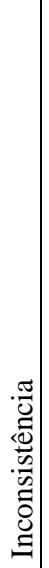 & 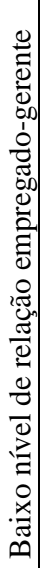 & 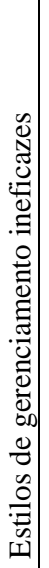 & 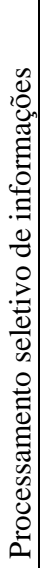 & 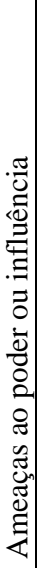 & 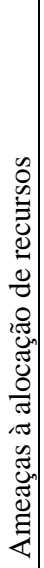 & 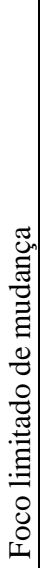 & 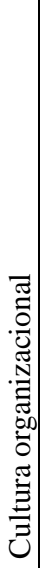 & 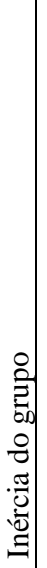 & 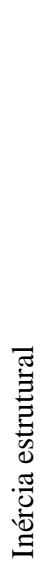 \\
\hline & $\stackrel{n}{\mathscr{\varepsilon}}$ & 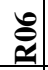 & $\hat{\tilde{\varepsilon}}$ & 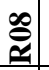 & $\stackrel{\vartheta}{\hat{\mathbf{q}}}$ & $\stackrel{\theta}{\underline{x}}$ & $\vec{z}$ & $\underline{\mathbf{v}}$ & $\frac{m}{\underline{a}}$ & $\underset{\Xi}{ \pm}$ & $\frac{10}{\underline{a}}$ & $\underset{a}{a}$ & $\underline{\underline{a}}$ & $\stackrel{\infty}{a}$ & $\underline{\underline{a}}$ & త્ઞ & 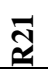 \\
\hline O01 & & & $\mathrm{x}$ & & & $\mathrm{x}$ & & & & & & & & & & & \\
\hline $\mathrm{O02}$ & & & $\mathrm{x}$ & & & $\mathrm{x}$ & & & & & & & $\mathrm{x}$ & & & & \\
\hline O03 & & & $\mathrm{X}$ & $\mathrm{X}$ & & $\mathrm{X}$ & & & & & & & $\mathrm{X}$ & $\mathrm{X}$ & & & \\
\hline 005 & $\mathrm{x}$ & & $\mathrm{X}$ & & & $\mathrm{X}$ & & & & $\mathrm{X}$ & & & & & & & \\
\hline 006 & & & $\mathrm{X}$ & & & $\mathrm{X}$ & & & & & & & & & & & \\
\hline 007 & & & $\mathrm{x}$ & & & $\mathrm{x}$ & & & & & & & $\mathrm{x}$ & & & & \\
\hline O09 & & $\mathrm{x}$ & & & & $\mathrm{x}$ & & & & & & & & & $\mathrm{x}$ & & $\mathrm{X}$ \\
\hline 012 & & & & & & $\mathrm{X}$ & & & & & & & & & $\mathrm{x}$ & & \\
\hline $\mathrm{O20}$ & & $\mathrm{x}$ & & & $\mathrm{x}$ & $\mathrm{X}$ & $\mathrm{x}$ & & $\mathrm{x}$ & $\mathrm{X}$ & & & & & & & \\
\hline $\mathbf{0 2 3}$ & & & $\mathrm{X}$ & & & $\mathrm{X}$ & $\mathrm{X}$ & & $\mathrm{X}$ & & & & & & & & \\
\hline O24 & & & $\mathrm{x}$ & & & $\mathrm{X}$ & & & $\mathrm{X}$ & & & & $\mathrm{x}$ & & & & \\
\hline $\mathbf{0 2 5}$ & & & $\mathrm{x}$ & & & & & & & & & & & & & & $\mathrm{X}$ \\
\hline O37 & & & & & & & & & & $\mathrm{X}$ & & & & & & & \\
\hline 044 & & & & & & $\mathrm{x}$ & & & $\mathrm{X}$ & $\mathrm{X}$ & & & & & & & \\
\hline 045 & & & & & & & & & $\mathrm{x}$ & $\mathrm{x}$ & & & & & & & \\
\hline 046 & & & & & & & & & $\mathrm{X}$ & $\mathrm{x}$ & & & & & & & \\
\hline
\end{tabular}

Fonte: Autores.

Quanto à cultura organizacional, associada pelo item de obstáculo "cultura organizacional não suportada" (O9), pode resultar das restrições estruturais da empresa, associada ao item "inércia estrutural" (C21). Já o item de obstáculo "falta de consciência de risco dentro da organização" (O12), pode resultar da "falta de comprometimento do conselho e da alta direção (R10), ou seja, dos participantes da mudança, que seriam responsáveis por gerar o ambiente de necessidade para a investigação dos riscos. Esta fonte de resistência (R10) aparece como uma das mais importantes para implementação do GRE, tendo conexão direta com 12 itens críticos de obstáculo $(\mathrm{O} 1, \mathrm{O} 2, \mathrm{O} 3, \mathrm{O} 5, \mathrm{O} 6, \mathrm{O} 7, \mathrm{O} 9, \mathrm{O} 12, \mathrm{O} 20, \mathrm{O} 23, \mathrm{O} 24, \mathrm{O} 44)$, resultando em barreiras aos processos de levantamento de dados, recursos, técnicas, habilidades, cultura e estratégia.

O item de obstáculo (O20) apresenta associação aos itens "mal-entendido" (C06) e "má comunicação interna" (C09), caracterizando fontes de resistência na organização. Segundo Zhao, Low \& Hwang (2014), programas de treinamento em GRE podem ser um canal de comunicação, através do qual toda a equipe pode compreender melhor os aspectos relativos à filosofia, política e processos de GRE, bem como a aplicação de suas técnicas e ferramentas. 


\section{Discussão dos Resultados}

Objetivando avaliar os aspectos relacionados à implantação de um sistema de gerenciamento de riscos em Cingapura, Zhao, Low e Hwang (2014) adotaram o escore t acima de 3,00 para valores críticos e $p<0,05$. No entanto, esta pesquisa considerou o escore $\mathrm{t}$ acima de 4,00 e $p<0,05$, para a obtenção dos fatores críticos. Dessa forma, apesar de adotar diferentes critérios, 4 dos 6 facilitadores críticos deste estudo foram também considerados críticos pela pesquisa base (F07, F08, F14, F17).

O item facilitador "Requisitos legais e de conformidade regulamentar" aparece como crítico no Brasil, mas não em Cingapura, indicando a possibilidade de questões locais influenciarem nesse resultado, como ocorre com o excesso de burocracia no Brasil, visto que, segundo Quah (2018), em 2016, o governo de Cingapura foi considerado o mais eficaz em aspectos burocráticos, em um rank que contempla países como Dinamarca, Austrália, China, Japão, Hong Kong e Índia. Em uma escala que vai de $-2,5$ até $+2,5$, seu escore atingiu a marca de $+2,2$.

Seguindo esse mesmo referencial, a FIESP (2010) registrou que a burocracia é um dos maiores obstáculos para o crescimento econômico e para a expansão dos negócios no Brasil.

Para os osbstáculos críticos, dos 36 itens inicialmente contemplados por Zhao, Low e Hwang (2014), 12 obstáculos desta pesquisa também foram apontados como críticos pelo estudo de Cingapura (O1, O2, O3, O5, O6 O7, O9, O12, O20, O23, O24, O25) o que aponta para uma consistência crítica em ambas as pesquisas.

\section{Conclusões}

Esta pesquisa teve como propósito, interpretar a implementação do GRE em empresa de construção civil no Brasil, comparando os resultados as teorias da mudança organizacional, seguindo o modelo aplicado em pesquisa de Cingapura. Foram identificados fatores e obstáculos à implementação do GRE, sendo 36 obstáculos de Cingapura e 14 nacionais, além de 17 facilitadores extraídos de pesquisa de Cingapura e 5 nacionais.

Os resultados indicaram que 6 fatores facilitadores e 16 obstáculos tiveram influência significativa na implementação do GRE. Além disso, considerando a implementação do GRE em empresas de construção como uma mudança organizacional, este estudo interpretou os fatores críticos e obstáculos da perspectiva da mudança organizacional, comparando com resultados já obtidos em Cingapura. Especificamente, tanto a Teoria do E quanto a Teoria do $\mathrm{O}$ se mostraram válidas na implementação da GRE. Em complemento, 17 fontes de resistência à mudança organizacional foram associadas aos 16 obstáculos críticos para a implementação do GRE.

Por fim, foram comparados os fatores críticos em ambos os países, evidenciando que as questões de burocracia apontadas pelo item crítico "requisitos legais e de conformidade regulamentar", assumem maior protagonismo no Brasil, quando comparado aos resultados apresentados em Cingapura.

Quanto às limitações da pesquisa, apesar da amostra atender aos critérios estatísticos de forma suficientemente, maior parte dos respondentes está localizada no estado do Rio de Janeiro, o que remete a precauções em interpretações generalistas e extrapoladas em todo o território nacional.

Com isso, sugere-se que estudos futuros possam comparar os fatores que facilitam e dificultam a implementação da GRE entre si, sob análise fatorial multivariada, procurando observar quais grupos podem ser formados e se há correlação significante entre eles. Outra possibilidade seria levantamento e análise do nível de maturidade da gestão de risco nas empresas de construção do Brasil.

\section{Referências}

Beer, M., \& Nohria, N. (2000). Cracking the Code of Change. Harvard Business Review, p 62. 
Berenger Y., R., \& Justus N, A. (2016). Risk management in the construction industry: a new literature review. MATEC web of Conferences $\mathrm{p} 66$. Johannesburg: University of Johannesburg.

Bressian, C. L. (2004). Mudança Organizacional: Uma visão gerencial. Gestão de negócios.

Campos, K. C., \& Carvalho, H. R. (2007). Análise Estatística Multivariada: Uma aplicação na atividade agrícola irrigada do município de Guaiúba - CE. Revista de Economia da UEG, 107-124.

Ching, H. Y. (2011). Contribuição das boas práticas do mercado para a eficiência na gestão de risco corporativo. REBRAE. Revista Brasileira de Estratégia, Curitiba, 4(3), 257-273.

Costa, A., Felippe, M. F., \& Reis, G. (2015). Licenciamento Ambiental de Grandes Empreendimentos Minerários: Dos Alarmes que Ninguém Escuta à Tragédia no Rio Doce. Vale do Rio Doce: formação geo-histórica e questões atuais, 96-98.

Costa, G. D., Castro, S. R., \& Victor Hugo, G. D. (2006). Um Procedimento Inferencial para Análise Fatorial Utilizando As Técnicas Bootstrap e Jackknife: Construção de intervalos de confiança e testes de hipóteses. Pontifícia Universidade Católica do Rio de Janeiro - PUC-RIO - Tese.

Creswell, J. W. (2012). Qualitative Inquiry and Research Design: Choosing Among Five Approaches. Thousand Oaks, CA: Sage Publications.

Cruz, F. M., Arezes, P. F., \& Junior, B. B. (2018). Avaliação dos efeitos sinérgicos sobre os trabalhadores expostos a fatores de risco físico em simultâneo. Universidade do Minho - Escola de engenharia - Tese.

Fialho, K. E., Costa, H. N., Lima, S. H., \& Barros Neto, J. de (2014). Aspectos Econômicos da Construção Civil no Brasil. XV Encontro Nacional de Tecnologia do ambiente construído. Universidade Federal do Ceará, 1105-1114.

FIESP. (2010). Relatório Burocracia: Custos econômicos e propostas de combate. DECOMTEC - Área de competitividade.

Freitas, A. L., \& Rodrigues, S. G. (2005). A avaliação da confiabilidade de questionários: uma análise utilizando alfa de cronbach. XII SIMPEP.

Freitas, A. P. A. (2018). Metodologia de Gerenciamento de Riscos na Indústria da Construção Civil. UFF - Tese.

Gordon, L. A., Loeb, M. P., \& Tseng, C.-Y. (2009). Gestão de risco empresarial e desempenho da empresa: uma perspectiva de contingência. Journal of Accounting and Public Policy, 28, $301-327$.

Guimarães, P. R. (2008). Métodos Quantitativos EstatísticosIESDE Brasil.

Hair Jr., J. F., Black, W. C., Babin, B. J., \& Anderson, R. E. (2014). Multivariate Data Analysis. Pearson New International Edition.

Hundertmark, T., Silva, A. O., \& Shulman, J. A. (2008). Managing Capital Projects. TheMcKinseyQuarterly.

ISO 31000. (2018). Associação Brasileira de Normas Técnicas.

Ito, S. (2018). Pesquisa Maturidade do Processo de Gestão dos Riscos no Brasil. KPMG.

Medeiros, R. K., Júnior, M. A., Pinto, D. P., Vitor, A. F., Santos, V. E., \& Barichello, E. (2015). Modelo de validação de conteúdo de Pasquali nas pesquisas de Enfermagem. Artigo de Revisão, 127-135.

Miot, H. A. (2017). Avaliação da normalidade dos dados em estudos clínicos e experimentais. Editorial, Universidade Estadual Paulista - UNESP, Faculdade de Medicina de Botucatu, Departamento de Dermatologia e Radioterapia, Botucatu, São Paulo, Brasil

Mok, C. R. (1997). Practices, barriers, and benefits of risk management process in building services cost estimation. Construction Management and Economics, 161-175.

Neves, C. E., Rodrigues, I. A., \& Porto, M. C. (2013). Guia de gerenciamento de Riscos de obras rodoviárias. DNIT.

Oliveira, F. C., \& Piretti, T. P. (2016). Técnicas de Gestão de Riscos: Um modelo para aplicação na construção civil leve brasileira. Brasília: UNB.

Paulo, W. L., Fernandes, F. C., \& Silva, M. Z. (2017). Modelo de Otimização de Recursos Financeiros para o Gerenciamento de Riscos Empresariais. Sistemas e Gestão, 98-107.

PMBOK. (2017). Conhecimento em gerenciamento de projetos. PMI.

Quah, J. S. (2018). Why Singapore works: five secrets of Singapore's success. Emerald insight, 5-21.

Renault, B. Y., \& Agumba, J. N. (2016). Risk Management in the construction industry. IBCC.

Ruppenthal, J. E. (2013). Gerenciamento de Riscos. E-tec Brasil.

Santos Jhunior, R. d., Abib, G., Vilela, N. G., \& Stocker, F. (2018). Percepção de Risco no Contexto Prévio à Internacionalização: Um estudo de caso. Congresso internacional de Administração, Revista Gestão e Planejamento, 20, 90-109.

Silva, V. F., \& Soeiro, A. A. (2012). Análise de Risco na Construção - Guia de procedimentos para gestão. Universidade do Porto - Tese.

Sundaram KR, Dwivedi SN, \& Sreenivas V (2014). Medical Statistics: Principles and Methods. (2a ed.), Wolters Kluwer India.

TCU. (2018). Referencial básico de gestão de riscos. TCU (tribunal de contas da União), Secretaria Geral de Controle Externo (SEGECEX). 
Research, Society and Development, v. 10, n. 6, e10710615487, 2021

(CC BY 4.0) | ISSN 2525-3409 | DOI: http://dx.doi.org/10.33448/rsd-v10i6.15487

Teixeira, J. C., Kulejewski, J., Krzemiński , M., \& Zawistowski, J. (2011). Gestão do Risco na Construção. Biblioteca de Gestão da Construção.

Thomas, R. a. (2011). Reframing resistance to organizational change. Scandinavian Journal of Management, 27(3), 322-331.

Granadeiro Guimarães (2017). Tribunal Regional do Trabalho $3^{\mathrm{a}}$ Região Minas Gerais, Fonte: http://www.granadeiro.adv.br/clipping/jurisprudencia/2017/08/29/influencias-da-religiao-no-trabalho-profissao-espiritualidade-caminham-juntas.

Whitaker, P., \& Reuters. (2017). O GLOBO. Fonte: https://oglobo.globo.com/economia/em-uma-semana-crise-politica-tira-184-bilhoes-de-empresasbrasileiras-21385616.

Yirenkyi, A. B., \& Chileshe, F. N. (2015). An analysis of risk management in practice: the case of Ghana's construction industry. "melhoria da moral e da produtividade da equipe", "produto da qualidade requerida" e "percepção pública melhorada". Journal of Engineering Design and Technology, 240 - 259.

Zhao, \& Singhaputtangkul. (2016). Effects of firm characteristics on Enterprise Risk Management: Case Study of Chinese Construction Firms Operating in Singapore. Journal of Management in Engineering.

Zhao, H. \& L. (2015). Reducing Hindrances to Enterprise Risk Management Implementation in Construction Firms. Journal of Management in Engineering.

Zhao, Hwang \& Low. (2014). Investigating Enterprise Risk Management Maturity in Construction Firms. Journal of Management in Engineering.

Zhao, X., Hwang, B.-G., \& Low, S. P. (2014). Enterprise risk management implementation in construction firms: An organizational change perspective. Management Decision, Vol. 52 Iss 5,. $814-833$.

Zhao, X., Hwang, B.-G., ASCE, M., \& Low, S. P. (2014). Investigating Enterprise Risk Management Maturity in Construction Firms. Journal of Management in Engineering. 